\title{
Systemic Lupus Erythematosus Presenting as Thrombotic Thrombocytopenia Purpura
}

\author{
Filipa Silva ${ }^{\mathrm{a}, \mathrm{c}}$, Mariana Brandao ${ }^{\mathrm{b}}$, Afonso Esteves ${ }^{\mathrm{a}}$
}

\begin{abstract}
Thrombotic thrombocytopenic purpura (TTP) is an uncommon disorder characterized by the classic pentad of microangiopathic hemolytic anemia, thrombocytopenia, renal dysfunction, fever and neurologic abnormalities. It is associated with infections, malignancy, drugs and autoimmune diseases. TTP and systemic lupus erythematosus (SLE) rarely present simultaneously and generally constitute a diagnostic dilemma. We present a case of a 42-year-old patient with overlapping features of SLE and TTP, who was admitted to our hospital with complaints of generalized fatigue, epistaxis and bruises. The workup revealed microangiopathic hemolytic anemia (negative Coomb's test with schistocytes), and thrombocytopenia. The presence of clinical and immunological criteria made the diagnosis of SLE possible. Recognition of TTP in the setting of SLE is important, because the combination of both conditions carries a worse prognosis than SLE alone. Prompt treatment with plasmapheresis and aggressive immunosuppressive therapy is life saving.
\end{abstract}

Keywords: Systemic lupus erythematosus; Thrombotic thrombocytopenic purpura; ADAMTS-13; Plasmapheresis; Immunosuppressive therapy

\section{Introduction}

Thrombotic thrombocytopenic purpura (TTP) is a rare systemic microvascular occlusive disorder characterized by the classic pentad of thrombocytopenia, microangiopathic hemolytic anemia, neurological abnormalities, renal dysfunction and fever [1].

Manuscript accepted for publication November 17, 2016

${ }^{a}$ Internal Medicine Department, Centro Hospitalar de Sao Joao, Porto, Portugal

bMedical Oncology Department, Instituto Portugues de Oncologia, Porto, Portugal

${ }^{\mathrm{c} C o r r e s p o n d i n g ~ A u t h o r: ~ F i l i p a ~ S i l v a, ~ S e r v i c o ~ d e ~ M e d i c i n a ~ I n t e r n a, ~ C e n t r o ~}$ Hospitalar de Sao Joao, E.P.E.. Alameda Prof. Hernani Monteiro, 4200-319 Porto, Portugal. Email: filipadcs@gmail.com

doi: https://doi.org/10.14740/jmc2692w
TTP can be primary or secondary to drugs (such as quinine, chemotherapy and immunosuppressants), marrow or organ transplantation, malignant hypertension or autoimmune diseases such as systemic lupus erythematosus (SLE) and rheumatoid arthritis. SLE and TTP may precede each other by a variable period of time $[1,2]$.

Patients with SLE can have significantly reduced ADAMTS-13 activity ( $<5 \%$ of normal), due to the presence of IgG anti-ADAMTS13 antibodies, suggesting a possible common pathophysiology for these diseases. Despite that, TTP occurring in patients with SLE can be difficult to diagnose because of overlapping features of the two disorders and the presence of other potentially concomitant thrombotic microangiopathies [3].

We present a case of a 42-year-old male patient with simultaneous features of SLE and TTP to emphasize that the recognition of TTP in the setting of SLE is important, because the combination of conditions carries a worse prognosis than SLE alone and prompt acknowledgment is essential for appropriate treatment with plasmapheresis and aggressive immunosuppressive therapy $[2,4]$.

\section{Case Report}

A 42-year-old Caucasian man, employee in a storage, asymptomatic until 2 weeks prior to admission when he started complaints of generalized fatigue, epistaxis, bruising over the upper and lower extremities. He also referred photosensitivity and Raynaud's phenomenon and denied dyspnea, thoracic pain, gastrointestinal or urinary complaints. He was examined in his general practitioner, who requested laboratory blood analyses that revealed severe anemia and thrombocytopenia, and was transferred to our emergency department.

Eight years ago, the patient was evaluated in a rheumatology consult because of arthritis in metacarpophalangeal joints and proximal interphalangeal with morning stiffness. Immunological study showed ANA 1/160 with speckled pattern, rheumatoid factor positive $(41.3 \mathrm{IU} / \mathrm{mL})$, positive antiRNP, SSa and SSb. He was diagnosed with undifferentiated connective-tissue disease and after 4 years, he immigrated and lost follow-up.

On physical examination, he was alert and oriented, with pale skin and bruising on upper and lower extremities, had 
temperature of $37.3{ }^{\circ} \mathrm{C}$, blood pressure of $115 / 69 \mathrm{~mm} \mathrm{Hg}$, pulse of $61 / \mathrm{min}$, and respiration of $16 / \mathrm{min}$. Arthritis of the second and third metacarpophalangeal joints of right hand was noted. Cardiovascular and pulmonary examination was normal and his abdomen was soft without tenderness or hepatosplenomegaly on palpation.

The blood tests on admission showed anemia of $6.4 \mathrm{~g} /$ $\mathrm{dL}$ with normal $\mathrm{MCV}$ and high reticulocyte index, thrombocytopenia $\left(25,000 / \mathrm{mm}^{3}\right)$, high $\mathrm{LDH}(2,059 \mathrm{U} / \mathrm{L})$, haptoglobin $<6 \mathrm{mg} / \mathrm{dL}$, and indirect hyperbilirubinemia $(2.6 \mathrm{mg} / \mathrm{dL})$ with normal C-reactive protein. Coomb's test was negative, renal functional, electrolytes and coagulation profile was within the normal limits. Peripheral blood smear revealed schistocytes. Chest X-rays were normal and abdominal ultrasound showed moderate hepatosplenomegaly. He had a blood transfusion.

The presence of hemolytic commitment, norerosive polyarthritis and photosensitivity associated with an immunological study showing low titers of C3 and C4, ANA 1/640 with speckled pattern and positive anti-Sm, fulfilled the diagnosis criteria for SLE.

Treatment with plasmapharesis sessions and prednisolone $1 \mathrm{mg} / \mathrm{kg}$ was started. After 6 days, he improved clinically with normalization of platelet count and in 2 weeks, hemoglobin, liver enzymes and LDH were within normal values.

The patient was discharged thereafter on tapering dose of prednisolone, hydroxychloroquine and methotrexate $10 \mathrm{mg} /$ week and after 2 years of follow-up, he remains in disease remission.

\section{Discussion}

With this clinical case, the authors pretend to draw attention to an infrequent association between SLE and TTP, which constitutes a difficult diagnosis and requires early effective treatment and follow-up.

Moschcowitz described TTP in 1925 after the autopsy of a 16-year-old patient, who died from anemia and petechiae, which revealed disseminated microvascular thrombi. In 1966, Amorosi and Ultmann, after reviewing a number of cases, formulated the classical pentad of thrombocytopenia, microangiopathic hemolytic anemia, neurological symptoms, kidney failure and fever. In this series, mortality was very high and decreased dramatically from $90 \%$ to $8-25 \%$ with the institution of plasma exchange transfusion [5].

Patients with TTP have a deficiency in a protease, called ADAMTS-13, which normally cleaves large von Willebrand factor (vWF) multimers into smaller and less adhesive forms. These unusually large vWFs promote abnormal platelet aggregation, resulting in microvascular thrombosis and thrombocytopenia affecting numerous organs, such as peripheral blood, central nervous system and kidney. Autoimmune diseases such as SLE have low levels of ADAMTS-13, suggesting a possible common pathophysiology between these two entities [6, 7].

Because treatment of these two diseases is different, distinguishing between the diagnoses is essential to ensure optimal clinical outcome. Therapy mainly consists of plasma exchange in TTP and corticosteroids and immunosuppressants in SLE. However, this may be especially difficult because of overlapping features of the two disorders, like hemolytic anemia, thrombocytopenia, fever, neurologic and kidney involvement. The characteristics that favor the diagnosis of TTP are the presence of fragmented red blood cells and schizocytes (indicating microangiopathic hemolytic anemia), associated with a negative Coomb's test and thrombocytopenia.

TTP can present in approximately $2 \%$ of the patients with SLE [8]. In most cases, it appears in patients previously diagnosed and treated for SLE for several years, with a high SLE disease activity index score and renal involvement [7]. Being the initial manifestation of SLE, as in our patient, is extremely uncommon. Is still a doubt if patients that present with TTP should be screen with ANA.

Recognition of simultaneous appearance of both conditions diseases is important, since it carries a worse prognosis than SLE alone. Mortality rate has been reported to be about $30 \%$ and the cause for this may be the delay in the institution of plasma exchange therapy [3].

\section{Conclusion}

In brief, the implications of this case are noteworthy for several reasons. Although uncommon, TTP and SLE can present and occur simultaneously. A high level of suspicion for TTP and SLE is demanded in patients who present with hemolysis, low platelets and fever. It is essential to distinguish whether the patient has SLE, TTP or both diseases, since early diagnosis and aggressive management with plasma exchange and immunosuppression is life saving.

\section{Conflicts of Interest}

None.

\section{References}

1. Garcia Boyero R, Mas Esteve E, Mas Esteve M, Milla Perseguer MM, Marco Buades J, Beltran Fabregat J, Canigral Ferrando G, et al. Systemic lupus erythematosus and thrombotic thrombocytopenia purpura: a refractory case without lupus activity. Reumatol Clin. 2013;9(6):373375 .

2. George P, Das J, Pawar B, Kakkar N. Thrombotic thrombocytopenic purpura and systemic lupus erythematosus: successful management of a rare presentation. Indian $\mathrm{J}$ Crit Care Med. 2008;12(3):128-131.

3. Cheung WY. Thrombotic thrombocytopenic purpura and systemic lupus erythematosus - distinct entities or overlapping syndromes? Transfus Apher Sci. 2006;34(3):263266.

4. Chak WK, Lam DS, Lo WH, Hui CM, Wong SN. Thrombotic thrombocytopenic purpura as a rare complication in childhood systemic lupus erythematosus: case report and literature review. Hong Kong Med J. 2003;9(5):363-368. 
5. Hassan A, Mahmoud E, Mohammad T. A Case Report of Thrombotic Thrombocytopenic Purpura Associated with Systemic Lupus Erythematosus: Overlapping Features. American Journal of Medical Case Reports. 2014;2(10):206-213.

6. George JN, Vesely SK, James JA. Overlapping features of thrombotic thrombocytopenic purpura and systemic lupus erythematosus. South Med J. 2007;100(5):512-514.
7. Perez CA, Abdo N, Shrestha A, Santos ES. Systemic lupus erythematosus presenting as thrombotic thrombocytopenia purpura: how close is close enough? Case Rep Med. 2011;2011:267508.

8. Kwok SK, Ju JH, Cho CS, Kim HY, Park SH. Thrombotic thrombocytopenic purpura in systemic lupus erythematosus: risk factors and clinical outcome: a single centre study. Lupus. 2009;18(1):16-21. 